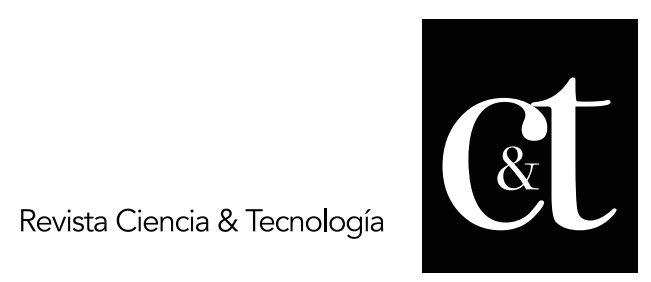

No. 32, 31 de octubre de 2021

ISSN impreso: 1390 - 6321

ISSN online: 2661 - 6734

\title{
Valoración de baba de cacao (mucílago) no utilizada en el cantón Quevedo - Ecuador
}

\section{Assessment of cocoa baba (mucilago) not used in Quevedo city - Ecuador}

\author{
Rossy Rodríguez Castro ${ }^{1}$ \\ rrodriguez@uteq.edu.ec \\ https://orcid.org/0000-0003-2193-6361 \\ Guiomar Posada Izquierdo ${ }^{2}$ \\ bt2poizg@uco.es \\ https://orcid.org/0000-0002-0158-3960 \\ Antonio Valero Díaz ${ }^{3}$ \\ bt2vadia@uco.es \\ https://orcid.org/0000-0001-8597-0740 \\ Emma Torres Navarrete 4 \\ etorres@uteq.edu.ec \\ https://orcid.org/0000-0002-9212-5593 \\ Yenny Torres Navarrete 5 \\ ytorres@uteq.edu.ec \\ https://orcid.org/0000-0003-3056-8708 \\ Raúl Díaz Ocampo 6 \\ rdiaz@uteq.edu.ec \\ https://orcid.org/0000-0002-8264-8614
}

Recibido: 28/6/2021, Aceptado: 28/9/2021

\section{RESUMEN}

La presente investigación está enfocada al desperdicio de mucílago fresco del cacao Theobroma cacao L., proveniente de las plantaciones de cacao de diversas zonas del Cantón Quevedo, ubicadas en la provincia de Los Ríos, perteneciente al Ecuador. La zona es tropical, húmeda con una temperatura media de 16 grados

\footnotetext{
${ }^{1}$ Magíster, Universidad Técnica Estatal de Quevedo, Ecuador/ Universidad de Córdoba, España

2 Doctora, Universidad de Córdoba, España

${ }^{3}$ Doctor, Universidad de Córdoba, España

${ }^{4}$ Magíster, Universidad Técnica Estatal de Quevedo, Ecuador

${ }^{5}$ Doctora, Universidad Técnica Estatal de Quevedo, Ecuador

${ }^{6}$ Doctor, Universidad Técnica Estatal de Quevedo, Ecuador
} 


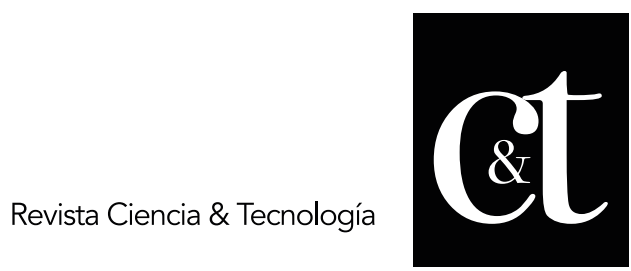

No. 32, 31 de octubre de 2021

ISSN impreso: 1390 - 6321

ISSN online: 2661 - 6734

centígrados. La variedad de cacao empleada para la investigación fue el Cacao Nacional; esto se analizó después de ser receptadas las mazorcas, extrayendo el mucílago al abrir las mazorcas y receptándola en tanques para mejor apreciación. Estas deben ser frutas maduras sin enfermedad y deben mantenerse a temperatura ambiente. La baba de cacao (el mucílago), siendo el principal componente para que se realice la fermentación de la almendra, ofrece excelentes propiedades organolépticas, asimismo brinda un aroma característico común de los productos a base de cacao y mucílago. Además, tiene alta concentración de azúcar y fibra; y pueden ser empleadas en la alimentación humana y en la industria alimenticia.

Actualmente se la utiliza de forma artesanal y a baja escala como materia prima fundamental para la elaboración de bebidas, jaleas, licores y diversos agricultores de la zona de Quevedo la emplean como base de herbicidas, por su característica adhesiva. Las muestras se obtuvieron de diversos cantones de la provincia de los Ríos, donde se analizaron 3 zonas específicamente Mocache, Buena Fe y Quevedo. Se realizó la recolección de 500 mazorcas por finca experimental de la variedad Nacional de Cacao. Fueron mazorcas maduras de corteza amarilla y sanas, con un peso promedio de $400 \pm 50$ gramos. Se obtuvo como resultados que aproximadamente el porcentaje que se desperdicia de mucílago en referencia a todo el fruto de cacao, incluyendo la corteza, es del $20 \%$ desaprovechado total del mucílago. A este se le puede dar la importancia a sus características, generando las condiciones para la elaboración, a base de valor agregado, de diversos productos para el consumo humano. Así, esto implica que se desaprovecha aproximadamente 40 litros de pulpa mucilaginosa que serían el resultado de 800 kilogramos de semillas frescas con mucílago de cacao.

Palabras clave: Baba, cacao, desperdicio, fermentación, mucílago

\begin{abstract}
This research is focused on the waste of fresh mucilage from Theobroma cacao $\mathrm{L}$. cacao, from cacao plantations in various areas of Cantón Quevedo, located in the province of Los Ríos, belonging to Ecuador. The area is tropical, humid with an average temperature of 16 degrees Celsius. The variety of cocoa used for the research was the National Cocoa; This was analyzed after the pods were received, extracting the mucilage when opening the pods and receiving it in tanks for better appreciation. These should be ripe, disease-free fruits and should be kept at room temperature. The cocoa slime (mucilage), being the main component for the fermentation of the almond to take place, offers excellent organoleptic properties, and provides a characteristic aroma common to cocoa and mucilage-based products. In addition, it has a high concentration of sugar and fiber; and they can be used in human food and in the food industry.
\end{abstract}

Currently, the artisanal way and a small scale are used as a fundamental raw material for the production of drinks, jellies, liquors and various farmers in the Quevedo area use it as a herbicide base, due to its adhesive characteristics. The samples were obtained from various cantons of the Los Ríos province, where 3 areas were specifically analyzed, Mocache, Buena Fe and Quevedo. The collection of 500 ears per experimental farm of the Nacional de Cacao variety was carried out. 


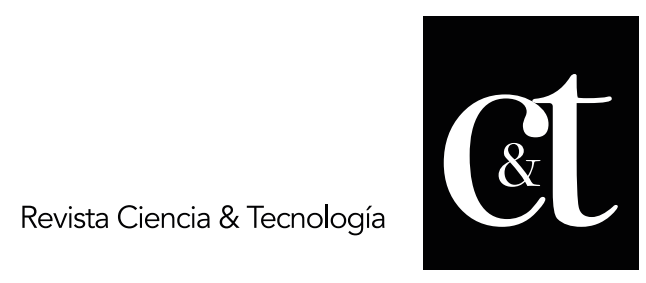

No. 32, 31 de octubre de 2021

ISSN impreso: 1390 - 6321

ISSN online: 2661 - 6734

They were mature ears with yellow bark and healthy, with an average weight of $400 \pm 50$ grams. The results were obtained that approximately the percentage of mucilage that is wasted in reference to the entire cocoa fruit, including the bark, is $20 \%$ of the total mucilage wasted.

This can be given importance to its characteristics, generating the conditions for the elaboration, a base of added value, of various products for human consumption.

Thus, this implies that approximately 40 liters of mucilaginous pulp is wasted, resulting in the result of 800 kilograms of fresh seeds with cocoa mucilage.

Keywords: slime, cocoa, waste, fermentation, mucilage

\section{Introducción}

En el mundo la producción de cacao se encuentra en mayor porcentaje en África con un $61 \%$, mientras que en América se produce un $21 \%$; a diferencia de Asia y Oceanía que llevan el $18 \%$. El Ecuador aporta un $50 \%$ de cacao fino de aroma; esto abarca 160.000 TM. En el año 2007 el país exportó 110.000 TM y tiende a aumentar su producción (Anecacao, 2012). El mucílago de cacao está conformado por un $80 \%$ de agua, de un 10 a $15 \%$ de glucosa y fructuosa, $1 \%$ de pectina y $1.5 \%$ de ácido cítrico. En principio el mucílago es simple pero, por la presencia de azúcares, proporciona excelentes características organolépticas (Braudeau, 2001).

El cacao es uno de los principales rubros agrícolas de exportación, que genera un ingreso representativo de divisas al país y, además, da reconocimiento internacional al Ecuador, por su calidad y aroma, único en el mundo; lo cual permite que se exporte prácticamente toda su producción.

El mucílago es una parte fundamental para el proceso de fermentación de la almendra, este a su vez debido a su contenido de azucares brinda un característico aroma (Puerari et al. 2012) el mucílago prácticamente es un rechazo del cacao, el cual puede ser aprovechado para la nutrición humana diaria, convirtiéndose en una materia prima base por su composición rica en proteínas (Luzuriaga, 2012). Además, hay distintas perspectivas según las diferentes clases de cacao producido, como dicen Amores, Suárez y Garzón, (2010) y Enríquez (2010).

Aunque la pulpa es necesaria para la fermentación, a menudo hay más pulpa de la necesaria. El exceso de la pulpa, que tiene un delicioso sabor tropical, ha sido utilizado para fabricar productos como jalea de cacao, alchol, vinagre, nata y pulpa procesada. 


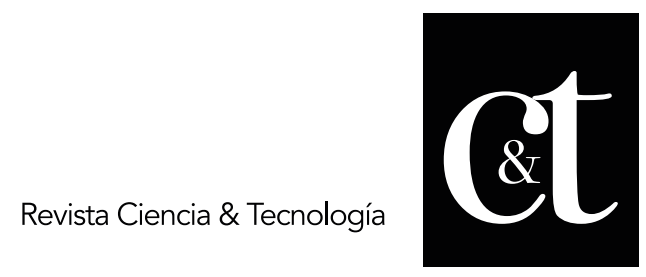

No. 32, 31 de octubre de 2021

ISSN impreso: 1390 - 6321

ISSN online: 2661 - 6734

Aproximadamente 40 litros de pulpa se puede obtener de 800 kilos de semillas frescas (Anecacao, 2012).

La presente investigación tuvo como finalidad valorar la cantidad de mucilago que se desperdicia, realizando un estudio en diversas zonas del cantón Quevedo.

\section{Método}

El presente trabajo se realizó en tres fincas experimentales de los cantones Quevedo, Buena Fe y Mocache, Provincia de Los Ríos. Ecuador, para cada tratamiento se utilizaron 5 repeticiones que consistieron en la recolección de 500 mazorcas de la variedad Nacional de cacao, fueron mazorcas maduras y sanas de tamaño promedio de cada zona, con un peso promedio de $400 \pm 50 \mathrm{gr}$.

Se realizó la recolección por la mañana del fruto de cacao, se registró la temperatura y los parámetros de calidad para la extracción del mucilago fresco, en el laboratorio de Bromatología, perteneciente a la Universidad Técnica Estatal de Quevedo, ubicada en el cantón Quevedo, provincia de Los Ríos, Ecuador.

El campo experimental está ubicado a $72 \mathrm{msnm}$, con una temperatura promedio anual de $25^{\circ} \mathrm{C}$ y 1000 milímetros de precipitación.

Se extrajo el mucilago mediante la tecnología de prensado, obteniendo mucilago limpio colocado en tanques de plástico e identificadas con cada tratamiento, Para evaluar las cantidades de mucilago se realizó un análisis de varianza de una vía y ante la existencia de diferencias significativas se utilizó el test LSD de rangos múltiples $(P<0,05)$. Para los análisis estadísticos fue utilizado el programa SPSS versión 19.0 .

Tipo de investigación

El proceso de la investigación se ejecutó de forma experimental y descriptive, evaluando las diferentes partes del cacao, teniendo en cuenta el mucílago principalmente; Almendras, placenta, cascara, etc., que están involucradas con cada uno de los tratamientos. Además se aplicó un diseño experimental aleatorio.

Investigación experimental

Esta investigación es de origen experimental, ya que se realizó con diferentes zonas estartégicas en pos de determiner la cantidad de mucílago de cacao durante el empleo del diseño.

Investigación descriptiva 
Este tipo de investigación se utilizó para la elaboración de los objetivos y para el marco teórico del trabajo, logrando determiner cada una de las características y propiedades con el fin de agrupar y ordenar cada una de las ideas a cumplir.

Métodos de investigación

Para la investigación se procedió con los siguientes métodos.

Inductivo

Se aplicó este tipo de método, ya que principalmenttte se comienza de un problema hacia la possible solución, la que permitirá al colectivo hallar una conexión para determiner la cantidad y tener en cuenta que se podrá desarrollar diversos alimentos con este subproducto.

\section{Resultados}

\section{Diseño}

\begin{tabular}{lll}
\hline Factores & Código & Descripción \\
\hline Cacao zonas & Zona 1 & Mocache \\
& Zona 2 & Buena Fe \\
& Zona 3 & Quevedo \\
\hline
\end{tabular}

Tabla 1. Peso de mucilago de cacao $(\mathrm{kg})$

\section{Repeticiones}

\begin{tabular}{lccccc}
\hline $\begin{array}{l}\text { Tratamien } \\
\text { to }\end{array}$ & R1 & R2 & R3 & R4 & R5 \\
\hline $\begin{array}{l}\text { Cacao Zona } \\
1\end{array}$ & 42 & 40 & 42 & 43 & 47 \\
$\begin{array}{l}\text { Cacao Zona } \\
2\end{array}$ & 49 & 43 & 41 & 41 & 45 \\
$\begin{array}{l}\text { Cacao Zona } \\
3\end{array}$ & 43 & 41 & 47 & 46 & 43 \\
\hline
\end{tabular}

Fuente: Elaboración propia 


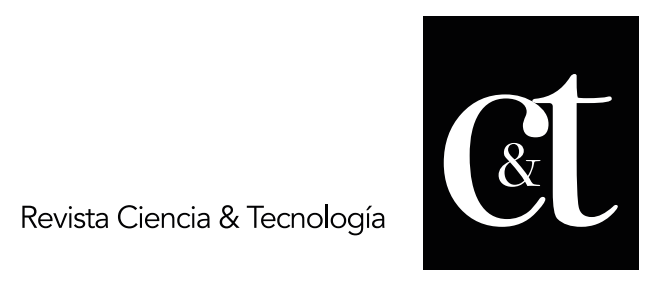

No. 32, 31 de octubre de 2021

ISSN impreso: 1390 - 6321

ISSN online: 2661 - 6734

Tabla 2. Resumen de datos.

\begin{tabular}{ccccc}
\hline Grupos & Cuenta & Suma & Promedio & Varianza \\
\hline Cacao & 5 & 214 & 42,8 & 6,7 \\
Zona 1 & 5 & 219 & 43,8 & 11,2 \\
Cacao & 5 & 220 & 44 & 6 \\
Zona 2 & 5 & & \\
Cacao & & &
\end{tabular}

\section{Discusión}

La tabla 1 muestra una generalización de los resultados dependiendo de las zonas analizadas para la estimación del mucílago de cacao. Se extrajo el mucílago de cacao por el método de prensado, se analizaron tres zonas como se representa en la tabla 1 , determinando una media (peso del mucílago). Además, es notorio que pueden y deben integrarse factores de explotación del producto cacao para la nutrición y actividad productiva (Kalvatchev, Garzaro y Guerra, 1998).

El resumen de datos expresado en la tabla 2, y mediante un análisis de varianza de una vía se determinó que no existe diferencias significativas, pero por su parte Arteaga (2013) confirma que aproximadamente de 40 litros de pulpa se pueden obtener de 800 kilos de semillas frescas; de la misma manera Villagómez et al (2013) manifiesta que el resultado proximal de líquido o exudado de es de $13.08 \mathrm{~L}$, realizado por dos métodos de extracción para el aprovechamiento del mucílago. Se debe realizar estudios adicionales para determinar la cantidad en los diferentes tiempos de almacenamiento.

\section{Conclusiones}

Uno de los elementos básicos es que en los propios sembradores y cosechadores dedicados al cultivo del cacao hace falta una valoración de los elementos nutrientes que existen en cada uno de los componentes del producto.

Son escasas, en los ámbitos de generación de productos y subproductos por parte de los agricultores de la zona, las gestiones de innovación para invertir esfuerzos en un plus a base del cacao. 


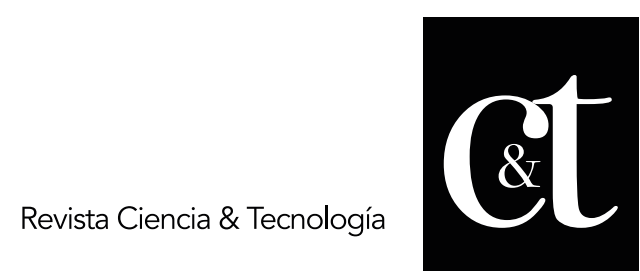

No. 32,31 de octubre de 2021

ISSN impreso: 1390 - 6321

ISSN online: 2661 - 6734

En las tres regiones visitadas, esto es, en Mocache, Buena Fe y Quevedo, existe una notoria falta de conciencia en lo concerniente a la utilización del mucílago de cacao, toda vez que eso atenta en contra del propio nivel de vida del sector agrícola: nuevos y continuos ingresos dejan de ser percibidos debido a este factor.

No ha habido tradicionalmente un seguimiento permanente a los distintos elementos del sector de la agricultura en lo que tienen que ver con la obtención de los subproductos del cacao. 


\section{Referencias bibliográficas}

Amores, F., Suárez, C. y Garzón, I. (2010). Producción intensiva de cacao nacional con sabor Arriba: Tecnología, presupuesto y rentabilidad.

Anecacao, (2012) Asociación Nacional de Exportadores de Cacao. Manual de cacao de pequeños productores. Programa de establecimiento de una estrategia de competitividad de la cadena de cacao fino y aroma del Ecuador. Guayaquil- Ec.

Braudeau (2001). El cacao. Técnicas agrícolas y producciones tropicales. Barcelona, España. Editorial Blumé. p 297

Enríquez, G. (2010). Cacao Orgánico: guía para productores ecuatorianos, Ecuador, INIAP.

Kalvatchev, Z., Garzaro, D. y Guerra, F. (1998). Theobroma cacao L.: Un nuevo enfoque para nutrición y salud. Revista agroalimentaria Vol. 4, No. 6, 1998 , págs. $23-25$

Puerari, C., Magallanes, T., \& Schwan, F. (2012). New cocoa pulp-based kefir beverages: Microbiological, chemical composition and sensory analysis. FRIN, 48, 634-640. http://doi.org/10.1016/j.foodres.2012.06.005.

Luzuriaga (2012). Extracción y aprovechamiento del mucilago de cacao (Theobroma cacao L.) como materia prima en la elaboración de vino. Disponible en:http://repositorio.ute.edu.ec/bitstream/ 123456789/14883/1/ 47745_1.

Arteaga Y (2013). Estudio del desperdicio del mucílago de cacao en el cantón Naranjal (provincia del Guayas). Revista ECA Sinergia. Facultad de Ciencias Administrativas y Económicas. U.T.M. Año 4 Vol. 4. p. 49.

Villagómez, F. Argüello (2013). Optimización y aprovechamiento del residuo (exudado del mucílago) de la almendra fresca del cacao (theobroma cacao I.) ccn51 en la elaboración de vinagre. Tsafiqui - Revista de Investigación Científica UTE, N4 